\title{
Effects of the presence of a pediatric intensivist on treatment in the pediatric intensive care unit
}

\author{
Jung Eun Kwon, Da Eun Roh, Yeo Hyang Kim \\ Division of Pediatric Cardiology, Kyungpook National University Children's Hospital and Department of Pediatrics, School of Medicine, Kyungpook National \\ University, Daegu, Korea
}

Background: There are few studies on the effect of intensivist staffing in pediatric intensive care units (PICUs) in Korea. We aimed to evaluate the effect of pediatric intensivist staffing on treatment outcomes in a Korean hospital PICU.

Methods: We analyzed two time periods according to pediatric intensivist staffing: period 1, between November 2015 to January 2017 (no intensivist staffing, $n=97$ ) and period 2, between February 2017 to February 2018 (intensivists staffing, $n=135$ ).

Results: Median age at admission was 5.4 years (range, $0.7-10.3$ years) in period 1 and 3.6 years (0.2-5.1 years) in period $2(\mathrm{P}=0.013)$. The bed occupancy rate decreased in period $2(75 \%$; $73 \%-88 \%)$ compared to period $1(89 \% ; 81 \%-94 \% ; P=0.015)$. However, the monthly bed turnover rate increased in period $2(2.2 \% ; 1.9 \%-2.7 \%)$ compared to period $1(1.5 \%, 1.1 \%$ $1.7 \% ; P=0.005)$. In both periods, patients with chronic neurologic illness were the most common. Patients with cardiovascular problems were more prevalent in period 2 than period 1 $(P=0.008)$. Daytime admission occurred more frequently in period 2 than period 1 (63\% vs. $39 \%, P<0.001)$. The length of PICU stay, parameters related with mechanical ventilation and tracheostomy, and pediatric Sequential Organ Failure Assessment score were not different between periods. Sudden cardiopulmonary resuscitations occurred in two cases during period 1 , but no case occurred during period 2.

Conclusions: Pediatric intensivist staffing in the PICU may affect efficient ICU operations.

Key Words: cardiopulmonary resuscitation; child; critical care; intensive care units

\section{INTRODUCTION}

The pediatric intensive care unit (PICU) is essential for the early detection and treatment of serious life-threatening conditions in severely ill infants, children, and adolescents. To cope with emergencies, adequate personnel and resources such as pediatric critical care specialists, nurses, and coordinators are required to ensure that established systems and protocols are fulfilled. The need for intensivists in the ICU is steadily increasing with the publication of studies showing that patient survival rates increase when intensivists are present [1,2]. The Leapfrog Group and American College of Critical Care Medicine developed guidelines in 1998 and 2003 that recommended ICU intensivist staffing. These staffing recommendations included not only normal work hour intensivist staffing but also the need for intensivists' immediate response to critical patients transferred to ICUs at other times [3-5]. In 2008, the Ko-

\section{Original Article}

Received: November 6, 2019

Revised: April 11, 2020

Accepted: April 16, 2020

Corresponding author Yeo Hyang Kim

Department of Pediatrics, School of Medicine, Kyungpook National University, 680 Gukchaebosang-ro, Jung-gu, Daegu 41944, Korea

Tel: +82-53-200-2747

Fax: +82-53-425-6683

E-mail:kimyhmd@knu.ac.kr

Copyright (@) 2020 The Korean Society of Critical Care Medicine

This is an Open Access article distributed under the terms of Creative Attributions Non-Commercial License (https:// creativecommons.org/li-censes/by-nc/4.0/) which permits unrestricted noncommercial use, distribution, and reproduction in any medium, provided the original work is properly cited. 
rean Society of Critical Care Medicine introduced a detailed system concerning critical care personnel needs. This system also stipulates that a critical care specialist may manage the ICU [6].

One study reported that, when an intensivist was present in the PICU, survival rate improved and better outcomes were reported for patients with diseases of different severity [7]. In 1985, the first PICU opened in the Seoul National University Children's Hospital. Currently, a total of 12 Korean hospitals operate PICUs and employ a total of 28 intensivists [8]. However, there are no data on the types of interventions performed by intensivists in the PICU and on the relationship between intensivist staffing and patients' prognoses in Korea. This study is the first report to evaluate the impacts of intensivist staffing in a Korean PICU. The purpose of this study was to investigate the differences in patients' characteristics and prognoses before and after intensivist staffing was implemented in the PICU of a single tertiary children's hospital in Korea.

\section{MATERIALS AND METHODS}

\section{Study Materials and Data Collection}

Patients who were admitted to the PICU of Kyungpook National University Children's Hospital (KNUCH) from November 2015 to February 2018 were included in the present study. The KNUCH opened in September 2013 with 100 beds. The PICU has five beds (including one isolation room) which are for both medical and surgical patients, including cardiac surgery. There is no separate pediatric cardiac ICU. The number of beds did not change from November 2015 to February 2018. Since February 2017, two intensivists have been working in the PICU, which has been operated as a closed and high-intensity ICU. Closed and high-intensity ICU means that the intensivist makes all clinical decisions for the patients from admission and treatment to discharge [6]. Intensivists worked during daytime hours (8:00 AM-8:00 PM) on weekdays and were on-call to work at any time needed on weekends, holidays, and nights. Since February 2017, a pediatric rapid response system has been assembled in the KNUCH, and two intensivists and one nurse participate in the pediatric rapid response system.

The study period was divided into two periods: period 1 (November 2015-January 2017) when there was no intensivist staffing and period 2 (February 2017-February 2018) when intensivists were present. All patients' medical records were retrospectively reviewed, and the following data were documented: patient's age at the time of admission to the PICU, cause

\section{KEY MESSAGES}

- Pediatric intensivist staffing in the pediatric intensive care unit (PICU) did not significantly reduce the length of stay or improve the priority classification of admitted patients.

- Intensivist staffing in the PICU decreased the bed occupancy rate and increased the monthly bed turnover rate.

- No sudden cardiopulmonary resuscitation events occurred on the general wards when the PICU had intensivist staffing.

for PICU admission, known chronic illness, critical care priority level according to ICU admission criteria [9], pediatric Sequential Organ Failure Assessment (pSOFA) score [10], length of stay, time spent on mechanical ventilation, course in hospital, and any cardiopulmonary resuscitation (CPR) events.

Admissions were divided into four groups according to the Society of Critical Care Medicine ICU admission prioritization model [9]. The priority 1 group included patients who required life support for organ failure, intensive monitoring, and therapies that are only available in the ICU environment. The priority 2 group included patients who required active monitoring and immediate treatment at any time with no limits on the extent of the therapy. The priority 3 group included those who could be managed at a lower level of care than the ICU. Patients in the priority 4 group were those with low or no probability of recovery/survival. The time of admission to the PICU was divided into daytime (8:00 AM-6:00 PM) and night time (remaining hours), for all weekdays, weekends, and holidays.

The pSOFA score [10] was calculated for every patient admitted to the PICU. The pSOFA score is calculated based on dysfunction by organ system, such as the respiratory, coagulation, hepatobiliary, cardiovascular, neurologic, and renal systems; the score ranges from a minimum of 0 to a maximum of 24 . The pSOFA score was measured daily until the 7 th day of admission. The initial score was defined as the first score on the first day of admission, and the peak score was defined as the highest score within the 7 days after PICU admission.

The primary outcome was to evaluate the patient's progress including length of the ICU stay, ventilator application, and mortality. The secondary outcome was to analyze operation of the ICU such as bed occupancy rate and bed turnover rate. The bed occupancy rate was calculated by dividing the average daily census by the number of beds. The bed turnover rate was calculated by dividing the number of discharges by the number of beds [11]. 


\section{Statistical Analysis}

All statistical analyses were performed using IBM SPSS ver. 23.0 (IBM Corp., Armonk, NY, USA). The values of variables are expressed as frequency or median and range. Because of non-normal distributions of the variables, we used the MannWhitney U-test to compare the two groups. A logistic regression was used to study the relationships among three major causes for admission. Analysis of covariance and CochranMantel-Haenszel tests with adjustment for pSOFA were performed to compare the difference of outcomes between period 1 and period 2 including death, expected or unexpected CPR, duration of PICU stay, and ventilation. A P-value of $<0.05$ was considered to be statistically significant.

\section{Ethics Statement}

The Institutional Review Board of Kyungpook National University Chilgok Hospital reviewed and approved the protocol for this study (IRB No. 2018-10-009), and waived the requirement for informed consent.

\section{RESULTS}

\section{Patient Characteristics}

During period 1, 97 patients were admitted to the PICU; during period 2, 135 patients were admitted. The age at admission was 5.4 years (range, 0.7-10.3 years) for period 1 and 3.6 years (range, $0.2-5.1$ years) for period $2(\mathrm{P}=0.013)$ (Table 1$)$. The bed occupancy rate decreased in period 2 (75\%; range, 73\%-88\%) compared to period 1 (89\%; range, $81 \%-94 \%$; $\mathrm{P}=$ $0.015)$. However, the monthly bed turnover rate increased in period 2 (2.2\%; range, $1.9 \%-2.7 \%$ ) compared to period 1 (1.5\%;

Table 1. Patient characteristics

\begin{tabular}{lccc}
\hline Variable & Period 1 $(n=97)$ & Period 2 $(n=135)$ & P-value \\
\hline Age at admission (yr) & $5.4(0.7-10.3)$ & $3.6(0.2-5.1)$ & 0.013 \\
Chronic illness & & & \\
$\quad$ Yes & $66(68)$ & $59(59)$ & 0.071 \\
$\quad$ None & $31(32)$ & $56(41)$ & \\
Time of admission & & & \\
$\quad$ Day & $38(39)$ & $85(63)$ & $<0.001$ \\
$\quad$ Night & $59(61)$ & $50(37)$ & \\
Monthly BOR (\%) & $89(81-94)$ & $75(73-88)$ & 0.015 \\
Monthly BTR (\%) & $1.5(1.1-1.7)$ & $2.2(1.9-2.7)$ & 0.005 \\
\hline
\end{tabular}

Values are presented as median (range) or number (\%). Period 1: between November 2015 to January 2017 (no intensivist staffing); period 2: between February 2017 to February 2018 (intensivists staffing). BOR: bed occupancy rate; BTR: bed turnover rate. range, $1.1 \%-1.7 \% ; \mathrm{P}=0.005$ ) (Table 1 ).

In terms of ICU admissions by priority level, 34 of patients (35\%) met the priority 1 criteria during period 1 , and $52(39 \%)$ were classified as priority 1 during period 2 . During period 1 , 60 of patients (62\%) were classified as priority 2 , and 82 of patients (61\%) were classified as priority 2 during period 2 . The number of patients who met the priority 1 and 2 criteria was not significantly different between period 1 and $2(\mathrm{P}=0.367)$. Three patients were classified as priority $4 \mathrm{~B}$ (and should not have been in the ICU) during period 1 and one patient was classified as priority $4 \mathrm{~B}$ in period 2 (Figure 1).

The causes for admission to the PICU were classified according to type of condition (Figure 2). When comparing the three major causes for admission during the two periods using logistic regression analysis, patients with cardiovascular problems were more prevalent in period 2 than period $1(\mathrm{P}=0.008)$. Of patients admitted to the PICU, 66 (68\%) had chronic illness in period 1 and $79(59 \%)$ in period $2(\mathrm{P}=0.139)$. In both periods, patients with chronic neurologic illness were the most common (Table 1, Figure 3). When comparing time of admission to the PICU, 38 of patients (39\%) in period 1 were admitted to the PICU during the daytime compared to $85(63 \%)$ in period $2(\mathrm{P}<0.001)$ (Table 1$)$.

\section{Hospitalization}

The length of PICU stay, parameters related with mechanical

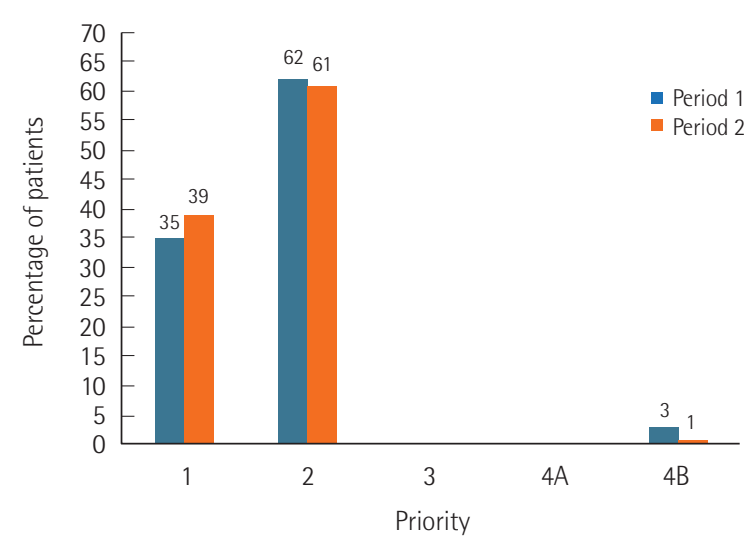

Figure 1. Percentage of admitted patients as per priority. Period 1: between November 2015 to January 2017 (no intensivist staffing); period 2: between February 2017 to February 2018 (intensivists staffing); Priority 1, patients who required life support for organ failure, intensive monitoring, and therapies that are only available in the intensive care unit (ICU) environment; priority 2, patients who required active monitoring and immediate treatment at any time with no limits on the extent of the therapy; priority 3, patients who could be managed at a lower level of care than the ICU; priority 4, patients with low or no probability of recovery/ survival. 


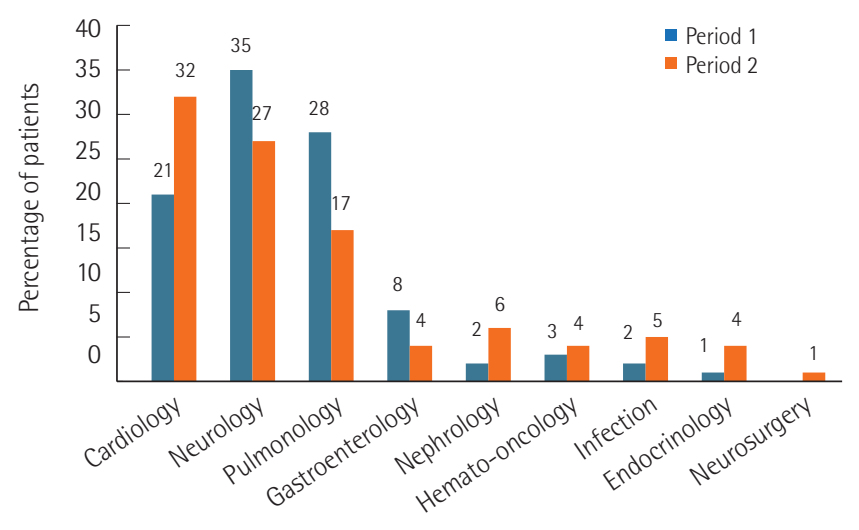

Figure 2. Cause for admission to pediatric intensive care unit (PICU). The three major causes for PICU admission during the two periods were cardiovascular, neurologic, and pulmonary problems. Period 1: between November 2015 to January 2017 (no intensivist staffing); period 2: between February 2017 to February 2018 (intensivists staffing).

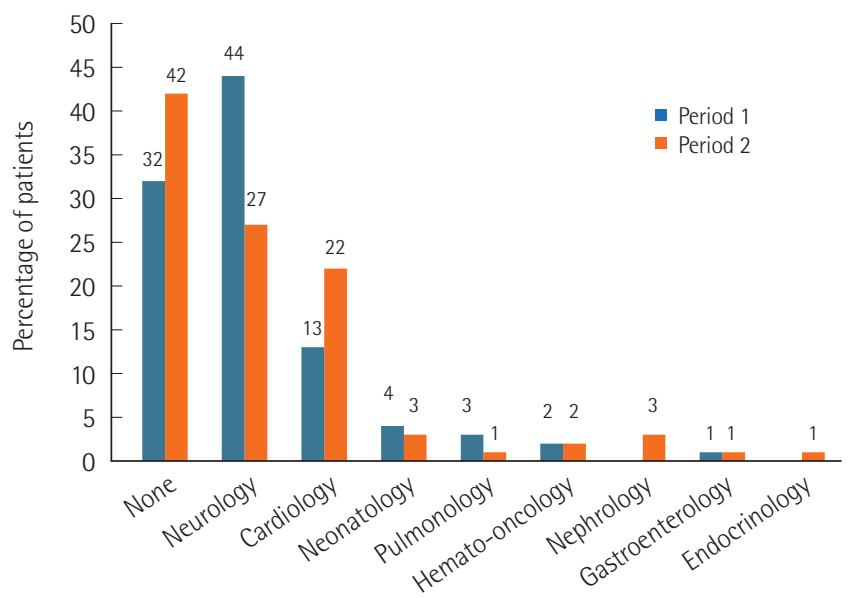

Figure 3. Classification of chronic illness. In both periods, patients with chronic neurologic illness were the most common. Period 1: between November 2015 to January 2017 (no intensivist staffing): period 2: between February 2017 to February 2018 (intensivists staffing).

Table 2. Hospitalization and prognosis

\begin{tabular}{|c|c|c|c|c|}
\hline Variable & Period $1(n=97)$ & Period $2(n=135)$ & P-value & Adjusted P-value ${ }^{a}$ \\
\hline \multicolumn{5}{|l|}{ pSOFA score } \\
\hline Initial & $3.0(1.0-6.5)$ & $3.0(2.0-6.0)$ & 0.529 & \\
\hline Peak & $6.0(2.0-11.0)$ & $4.5(2.0-7.8)$ & 0.173 & \\
\hline Length of PICU stay (day) & $3.0(2.0-17.0)$ & $4.0(2.0-14.0)$ & 0.367 & 0.755 \\
\hline Ventilator application & $43(44)$ & $56(41)$ & 0.688 & 0.756 \\
\hline Length of ventilator application (day) & $14.0(2.0-30.0)$ & $9.5(2.0-21.5)$ & 0.424 & 0.231 \\
\hline Application of ventilator for more than 30 days & $11(26)$ & $10(18)$ & 0.241 & 0.454 \\
\hline Length of ventilator application for more than 30 days (day) & $36.0(33.0-92.0)$ & $47.5(36.2-63.8)$ & 0.725 & \\
\hline Tracheostomy & 3 & 9 & 0.169 & \\
\hline Duration from PICU admission to tracheostomy (day) & $16(13-35)$ & $22(14-30)$ & & \\
\hline Death & $14(14)$ & $16(12)$ & 0.563 & 0.346 \\
\hline \multicolumn{5}{|l|}{ CPR } \\
\hline Expected & 4 & 6 & 0.934 & 0.812 \\
\hline Unexpected & 2 & 0 & 0.418 & 0.285 \\
\hline
\end{tabular}

Values are presented as median (range) or number (\%). Period 1: between November 2015 to January 2017 (no intensivist staffing); period 2: between February 2017 to February 2018 (intensivists staffing).

pSOFA: pediatric Sequential Organ Failure Assessment; PICU: pediatric intensive care unit; CPR: cardiopulmonary resuscitation.

astatistics value corrected with pSOFA.

ventilation and tracheostomy, and pSOFA score are shown in Table 2. Regarding procedures performed in the PICU, five patients in period 2 needed continuous renal replacement therapy (CRRT), and no patient needed this procedure during period 1 . After admission to the PICU, tracheostomy was performed in three patients in period 1 and nine patients in period $2(\mathrm{P}=0.169)$. All tracheostomy patients in period 1 and sev- en tracheostomy patients (78\%) in period 2 had neurologic and genetic problems. The median duration from PICU admission to tracheostomy was 16 days (range, 13-35 days) in period 1 and 22 days (range, 14-30 days) in period 2. A home ventilator was required for one patient in period 1 and nine patients in period 2 .

The initial pSOFA score on the first day of PICU admission 
was 3.0 (range, 1.0-6.5) in period 1 and 3.0 (range 2.0-6.0) in period $2(\mathrm{P}=0.529)$. The peak pSOFA score during PICU stay was 6.0 (range, 2.0-11.0) in period 1 and 4.5 (range, 2.0-7.8) in period $2(\mathrm{P}=0.173)$. The peak score for deceased and surviving patients were not different between period 1 and 2 (13.9 \pm 3.4 vs. $14.0 \pm 3.5, \mathrm{P}=1.000 ; 5.5 \pm 4.4$ vs. $5.3 \pm 3.8$, respectively, $\mathrm{P}=0.961$ ). Fourteen patients (14\%) died in period 1 and $16(12 \%)$ in period 2; the difference was not significant $(\mathrm{P}=0.563)$. In the PICU, there were two unexpected, sudden CPR cases in period 1 , both of which were caused by acute respiratory failure. There were no CPR cases in period 2. On the general wards, there were nine CPR cases in period 1 and none in period 2.

\section{DISCUSSION}

Although we did not find significant differences in survival outcomes between the two periods we studied (with and without intensivist staffing), intensivist staffing in the PICU resulted in better bed occupancy and turnover rates due to more efficient use of the limited beds in the unit. These outcomes were related to the system operating as a closed and high-intensity ICU. PICU intensivists are able to make informed and appropriate admission (using admission criteria) and discharge decisions. This system of operation resulted in an increase in the number of patients admitted as well as improvement of occupancy and turnover rates. The decision to perform tracheostomy is an example of how intensivists improved PICU operating efficiency. The intensivists chose tracheostomy when no intensive treatments other than assisted respiration were needed. The intensivists promptly recognized when a patient could be safely transferred to a ward while using a home ventilator or high flow nasal cannula thereby shortening the average length of PICU stay.

Cardiovascular, neurologic, and respiratory problems were the main causes of admission to the PICU. In particular, patients with cardiovascular problems were hospitalized more in period 2 than in period 1 . A pediatric cardiologist has been on staff at the Children's Hospital since September 2016. The presence of the pediatric cardiologist may have been responsible for the increased number of patients admitted with heart disease. In addition, our PICU is a mixed ICU for both medical and surgical patients without a separate pediatric cardiac ICU.

The intensivist staffing affected the transfer timing in the PICU. We found that more patients were transferred into the PICU during daytime hours with intensivist staffing. An important point is that intensivists participated in the pediatric rapid response system optimized for screening and treatment of more serious patients. Patients who were likely to be admitted to the PICU were continuously monitored by intensivists using the pediatric rapid response system and were admitted to the PICU before their condition deteriorated.

The PICU intensivists have also influenced the type of procedures that are performed in the PICU. An example of this is the intensivist's capability to perform a catheter insertion for CRRT. Medical staff, including nurses, underwent repeated training to ensure a working environment in which the implementation of CRRT was supported. As mentioned above, performance of tracheostomies is also within an intensivist's area of expertise. Tracheostomy was performed only three times in period 1, but in period 2 there were 19 tracheostomies. The applications of home ventilators also increased.

Several studies have shown that intensivist staffing in the ICU improved the survival rate, decreased the length of stay, and significantly decreased ICU or hospital mortality $[1,2,6$, 12-14]. In addition, a previous study reported that the average number of days on mechanical ventilation decreased and the number of hospitalized patients in the ICU decreased with the intensivist system even though mortality did not significantly decrease [15]. However, in the present study, the effects of the PICU intensivist staffing on survival rate, length of PICU stay, and ICU or hospital mortality were not statistically significant.

Pronovost et al. [1] compared treatment outcomes according to the type of ICU and showed a significant reduction in mortality in high-intensity intensive care units compared to low-intensity intensive care units. Additionally, the prognosis of patients improved in hospitals with 24-hour coverage by intensivists [2]. In contrast, another previous study showed that there were no significant differences in treatment outcomes in an ICU with the high-intensity night time intensivist staffing model [16]. The KNUCH PICU followed the high-intensity daytime staffing model. Two sudden CPR events due to acute respiratory failure occurred in period 1 , but none occurred in period 2. We believe that this was related to the close monitoring of PICU patients' conditions by intensivists during period 2. Their early assessments of inadequate respiratory support prevented any respiratory failures.

A limitation of the present study is the retrospective study design, small sample size and, a relatively short period. Also, data were acquired from a single tertiary children's hospital in Korea. During the study period, two intensivists were working in the PICU. This number varies depending on region and institution, limiting our ability to generalize the study results. In conclusion, pediatric intensivist staffing in the PICU may affect efficient ICU operations. However, further study is needed to determine 
PICU intensivist staffing impact on patients' outcomes.

\section{CONFLICT OF INTEREST}

No potential conflict of interest relevant to this article was reported.

\section{ORCID}

Jung Eun Kwon

Da Eun Roh

Yeo Hyang Kim https://orcid.org/0000-0001-6608-3089

https://orcid.org/0000-0001-8932-2505

https://orcid.org/0000-0002-1631-574X

\section{AUTHOR CONTRIBUTIONS}

Conceptualization: YHK. Data curation: JEK, DER. Formal analysis: JEK. Methodology: YHK. Project administration: YHK. Visualization: JEK. Writing-original draft: YHK. Writing-review \& editing: YHK.

\section{REFERENCES}

1. Pronovost PJ, Angus DC, Dorman T, Robinson KA, Dremsizov TT, Young TL. Physician staffing patterns and clinical outcomes in critically ill patients: a systematic review. JAMA 2002;288: 2151-62.

2. Wilcox ME, Chong CA, Niven DJ, Rubenfeld GD, Rowan KM, Wunsch $\mathrm{H}$, et al. Do intensivist staffing patterns influence hospital mortality following ICU admission? A systematic review and meta-analyses. Crit Care Med 2013;41:2253-74.

3. Milstein A, Galvin RS, Delbanco SF, Salber P, Buck CR Jr. Improving the safety of health care: the leapfrog initiative. Eff Clin Pract 2000;3:313-6.

4. Gajic O, Afessa B. Physician staffing models and patient safety in the ICU. Chest 2009;135:1038-44.

5. Haupt MT, Bekes CE, Brilli RJ, Carl LC, Gray AW, Jastremski MS, et al. Guidelines on critical care services and personnel: recommendations based on a system of categorization of three levels of care. Crit Care Med 2003;31:2677-83.
6. Park S, Suh GY. Intensivist physician staffing in intensive care units. Korean J Crit Care Med 2013;28:1-9.

7. Goh AY, Lum LC, Abdel-Latif ME. Impact of 24 hour critical care physician staffing on case-mix adjusted mortality in paediatric intensive care. Lancet 2001;357:445-6.

8. Yoon JS, Jhang WK, Choi YH, Lee B, Kim YH, Cho HJ, et al. Current status of pediatric critical care in Korea: results of 2015 national survey. J Korean Med Sci 2018;33:e308.

9. Nates JL, Nunnally M, Kleinpell R, Blosser S, Goldner J, Birriel $\mathrm{B}$, et al. ICU admission, discharge, and triage guidelines: a framework to enhance clinical operations, development of institutional policies, and further research. Crit Care Med 2016; 44:1553-602.

10. Matics TJ, Sanchez-Pinto LN. Adaptation and validation of a pediatric Sequential Organ Failure Assessment Score and Evaluation of the Sepsis-3 definitions in critically ill children. JAMA Pediatr 2017;171:e172352.

11. Tripathi CB, Kumar R, Sharma RC, Agarwal R. Assessment of performance of services in a tertiary care Neuropsychiatric Institute using Pabon Lasso Model. Asian J Med Sci 2016;7:69-74.

12. Manthous CA, Amoateng-Adjepong Y, al-Kharrat T, Jacob B, Alnuaimat HM, Chatila W, et al. Effects of a medical intensivist on patient care in a community teaching hospital. Mayo Clin Proc 1997;72:391-9.

13. Hanson CW 3rd, Deutschman CS, Anderson HL 3rd, Reilly PM, Behringer EC, Schwab CW, et al. Effects of an organized critical care service on outcomes and resource utilization: a cohort study. Crit Care Med 1999;27:270-4.

14. Blunt MC, Burchett KR. Out-of-hours consultant cover and case-mix-adjusted mortality in intensive care. Lancet 2000; 356:735-6.

15. Wise KR, Akopov VA, Williams BR Jr, Ido MS, Leeper KV Jr, Dressler DD. Hospitalists and intensivists in the medical ICU: a prospective observational study comparing mortality and length of stay between two staffing models. J Hosp Med 2012; 7:183-9.

16. Wallace DJ, Angus DC, Barnato AE, Kramer AA, Kahn JM. Nighttime intensivist staffing and mortality among critically ill patients. N Engl J Med 2012;366:2093-101. 